\title{
miRNAs Expression and Role of Dicer on Podocyte Injury in PAN Nephrosis Rats
}

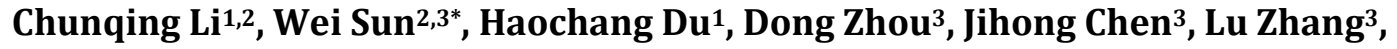 \\ Jiade Shao ${ }^{3}$ \\ ${ }^{1}$ Nephrology Department of Wuxi No. 3 Hospital, Wuxi, China \\ ${ }^{2}$ Discipline of Chinese and Western Integrative Medicine, Nanjing University of Chinese Medicine, Nanjing, China \\ ${ }^{3}$ Nephrology Department of Jiangsu Province Hospital of Traditional Chinese Medicine, Nanjing, China \\ Email: ${ }^{\text {jissunwei@163.com }}$
}

Received 1 January 2015; accepted 12 February 2015; published 16 February 2015

Copyright (C) 2015 by authors and Scientific Research Publishing Inc.

This work is licensed under the Creative Commons Attribution International License (CC BY).

http://creativecommons.org/licenses/by/4.0/

(c) () Open Access

\section{Abstract}

Objective: microRNAs (miRNAs) are regulatory RNAs that act as important players in diverse biologic and pathologic processes. Under circumstance as podocye-injury triggering proteinuria, which miRNAs are up-regulated or down-regulated? This experiment aims at detecting miRNAs changes in PAN nephrosis rats based on miRNA arrays and exploring the therapeutic targets of Leizhi capsule. Methods: Fifty male wistar rats were randomly divided into five groups, including control group, model group, leizhi capsule group, Tripterygium glucosides group, and valsartan group. PAN nephrosis models were made by jugular vein injection of PAN $(100 \mathrm{mg} / \mathrm{kg}$ body weight, dissolve in physiological saline), while control group rats were made by jugular vein injection of physiological saline with equal volume. Other groups rats had been given medicines by irrigating stomach once a day for ten days. Blood and urine samples were collected, and renal tissues were processed after rats being euthanasised. The $24 \mathrm{~h}$ urinary protein excretion and blood biochemistry parameters were measured by routine methods. The glomerular morphology and podocyte ultrastructure were observed with light microscopy and transmission electron microscopy respectively. miRNA expression profile was detected by Exiqon miRNA Array. Real time RT-PCR analysis for mature miRNAs was used to validate differentially expressed miRNAs. Results: 1) In day 3 - 5, model rats had decreased urine volume, ascites, malnutrition and wight loss. From day 7 to day 10, the nephrotic syndromes were worst in model rats, but which had no skin edema. Some rats died in serious ascites, the mortality is 3/10. 2) miRNA array detection shows 106 miRNAs up regulated and 62 miRNAs down regulated in PAN nephrosis rats. Fold change (model vs. control group) varies from 1.8 to 7.0. For leizhi capsule group and model sample, there are 90 miRNAs differentially expressed, with 65 miRNAs up and 25 miRNAs down. The most important finding in our research is the discovery of the specific miRNAs related to PAN nephrosis (rno-miR23a, rnomiR-24, rno-miR-30c and rno-miR-300-3p), which have been validated by Real time RT-PCR anal-

\footnotetext{
${ }^{*}$ Corresponding author.
} 
ysis. 3) Compared with control sample, immune fluorescence intensity of dicer, expression profile of nephrin, podocin and synaptopodin mRNA and protein decrease in PAN nephrosis rats. After treated with Leizhi Capsule, immune fluorescence intensity of the above molecules improved. Conclusion: 1) Characteristic miRNAs of PAN nephrosis were screening. Up-regulated miRNAs (rno-miR-23a, rno-miR-300-3p) may trigger podocyte injury and proteinuria, while down-regulated miRNAs (rno-miR-24, rno-miR-30c) may be protective factors by anti-apoptosis. 2) Dicer and these miRNAs (rno-miR-24, rno-miR-30c, rno-miR-23a) may be are probably key molecules therapeutic targets of Leizhi capsule.

\section{Keywords}

microRnAs, Podocyte Injury, Puromycin Aminonucleoside Nephrosis Model, Proteinuria, Leizhi Capsule

\section{Introduction}

microRNAs (miRNA) are a group of non-coding small RNA (approximately 22-nt) that are present in lower through higher organism and function to regulate gene expression by translation repression or transcript degradation of target genes. Dicer is a key enzyme in miRNA biosynthetic, by which pri-miRNAs are processed into approximately 22-nt mature microRNA [1] [2]. In addition, Dicer is involved in the immediate downstream effecter steps of this pathway, whereby it serves as an essential component of miRNA-containing catalytic enzyme complexes, such as the RNA-induced silencing complex. Thus, as a physical platform and a functional bridge, Dicer couples miRNA biogenesis to miRNA-mediated gene silence [3].

Proteinuria is a dangerous factor of chronic kidney disease (CKD). Podocyte injury is not only the key link of proteinuria, but also closely contacts with CKD progress. Some microRNAs may induce slit diaphragm (SD) molecules expression via triggering nephrin phosphorylation to reduce proteinuria [4]-[6]. As a key enzyme of miRNA biosynthesis, dicer probabaly have a role on proteinuria of CKD. Podocyte-selective deletion of dicer induces proteinuria and glomerulosclerosis [1] [7], also alter cytoskeletal dynamics and causes glomerular disease [1] [2] [8], leading to rapid glomerular and tubular injury [3] [9]. It is suggested that dicer and these miRNAs are probably key regulated targets. How dicer enzyme have effects on podocyte in PAN (puromycin aminonucleoside, PAN) nephrosis pathlogy? So the aim of our experiment is to explore how dicer has effects on podocyte apotosis and cytoskeleton.

\section{Methods}

Fifty male Wistar rats were divided into five groups, including: 1) control group, 2) PAN model group, 3) leizhi group, 4) Tripterygium glucosides (GTW) group, and 5) valsartan group.

PAN model rats were made by jugularly intravenous injecting with PAN (100 mg/kg) [10], while control group rats were made by intravenous injecting with physiological saline. After injection, all rats were feeding with clear water and food for ten days in metabolic cage.

By irrigation stomach once a day for ten days, all rats had been given medicines as follows:

1) Physiological saline (2 ml) for control group and model group;

2) Tripterygium glucosides (2 mg) dissolved in Erzhi (containing Ligustrum lucidum and Drought Ephraim grass) solusion (1 ml) for Leizhi group;

3) Tripterygium glucosides $1 \mathrm{mg} / \mathrm{kg} / \mathrm{d}$ for Tripterygium glucosides group;

4) Triptolid $2 \mathrm{mg} / 200 \mathrm{~g} / \mathrm{d}$ for triptolid group ;

5) Valsartan valsartan $1.5 \mathrm{mg} / 200 \mathrm{~g} / \mathrm{d}$ for valsartan group.

Urine was collected on alternate day, and blood sample were gathered before and after experiment. After all rats being euthanasised, kidneys and livers were removed for Electron microscopy, Immunofluorescence stain and RT-PCR and western bloting.

miRNA Array analysis has been accomplished by Shanghai KangChen Biological engineering company. miRNA expression profile was detected by Exiqon miRNA Array, including: prepare the RNA Sample and 
RNA Sample QC, miRNA labeling, miRNA array hybridization, miRNA array scanning and analysis. Real time RT-PCR analysis for mature miRNAs was used to validate 4 differentially expressed miRNAs between control and model group in microRNA microarray assays.

\section{Results}

PAN nephrosis rats were made successfully by jugular vein injection of PAN (100 mg/kg body weight). In day 5 , model rats were in low spirits, with decreased urine volume, ascites, malnutrition and weight loss. From day 7 to day 10, proteinueia for $24 \mathrm{~h}$ were worst in PAN model rats, but without skin edema, see Table 1. Some rats died of serious ascites, the mortality is $30 \%(3 / 10)$.

Morphologic changes in light microscope include epithelial cells degeneration renal tubular and transparent cast, but there are no obvious changes in glomerulus and renal interstitial. Podocyte processes effacement was obvious in model groups sample in electronic microscope. See in Figure 1.

miRNA array detection shows 106 miRNA up regulated and 62 miRNA down regulated in PAN nephrosis rats. Fold change (model vs. control group) vary from 1.8 to 7.0. For leizhi capsule high-dose group and model sample, there are 90 miRNA differentially expressed, with 65 up and 25 down. see Figure 2 and Figure 3.

The most important finding in our study is the discovery of the specific miRNA related to PAN nephrosis (rno-miR23a, rno-miR-24, rno-miR-30c and rno-miR-300-3p, which have been validated by Real time RT-PCR analysis. See Table 2.

Compared with control sample, Immune Fluorescence intensity of nephrin, podocin and synaptopodin reduced in model sample. See in Figure 3. In addition, apoptosised podocytes increase in PAN sample.

Table 1. Proteinuria for $24 \mathrm{~h}$ among each group.

\begin{tabular}{ccccc}
\hline Group & Dose $\mathrm{mg}^{\mathrm{kg}} \mathrm{kg}^{-1}$ & Pre-trial & $3 \mathrm{~d}$ & $9 \mathrm{~d}$ \\
\hline NS & - & $4.63 \pm 1.82$ & $4.43 \pm 1.05$ & $5.33 \pm 1.69$ \\
PNS & - & $5.29 \pm 2.68$ & $73.45 \pm 68.76^{1)}$ & $90.04 \pm 65.38^{1)}$ \\
Leizhi & 10 & $4.81 \pm 3.77$ & $38.24 \pm 13.41^{2)}$ & $28.23 \pm 15.26^{2)}$ \\
GTW & 10 & $6.82 \pm 4.09$ & $54.68 \pm 25.32^{2) 3}$ & $33.23 \pm 14.14^{2)}$ \\
Valsartan & 7.5 & $5.47 \pm 1.68$ & $44.46 \pm 17.66^{2)}$ & $23.09 \pm 5.41^{2)}$ \\
\hline
\end{tabular}

Compared with NS group, ${ }^{1)}$ representing $\mathrm{p}<0.05$, compared with PNS group, ${ }^{2}$ representing $\mathrm{p}<0.05$, compared with Leizhi group, ${ }^{3)}$ representing $\mathrm{p}<$ 0.05 .

Table 2. miRNAs expression by miRNA Array and realtime RT PCR. (a) miRNA expression (model versus NS). (b) miRNA expression (leizhi versus PAN).

(a)

\begin{tabular}{ccc}
\hline miRNA & miRNA Array & Realtime RT PCR \\
\hline rno-mir-24 & 0.32 & 0.312 \\
rno-mir-30c & 0.30 & 0.555 \\
rno-mir-23a & 3.23 & 2.472 \\
rno-mir-300-3p & 2.93 & 2.514 \\
\hline
\end{tabular}

(b)

\begin{tabular}{ccc}
\hline miRNA & miRNA Array & Realtime RT PCR \\
\hline rno-mir-24 & 3.49 & 0.312 \\
rno-mir-30c & 2.32 & 0.555 \\
rno-mir-23a & 0.50 & 2.472 \\
rno-mir-300-3p & 0.53 & 2.514 \\
\hline
\end{tabular}




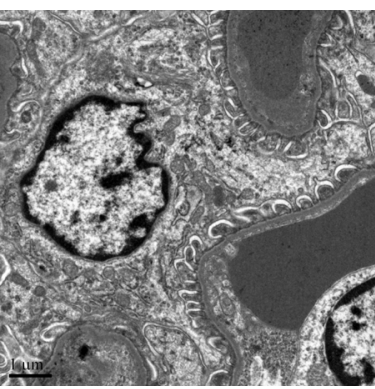

(a)

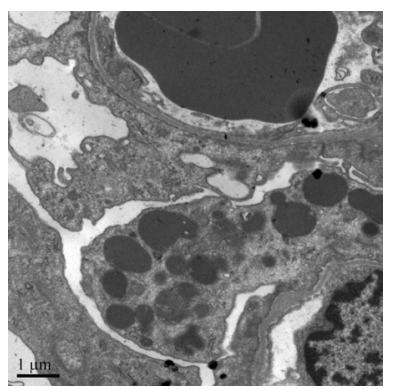

(b)

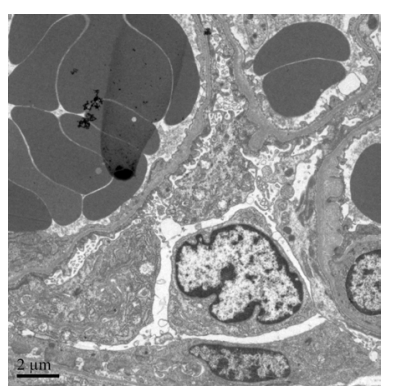

(c)

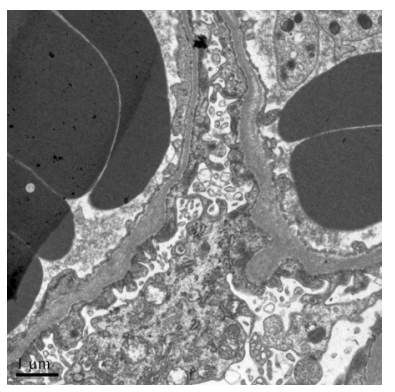

(d)

Figure 1. Change of foot process of glomerular podocyte in PAN rats. (a) NS, (b) PAN, (c) Leizhi, (d) GTW (8000×).

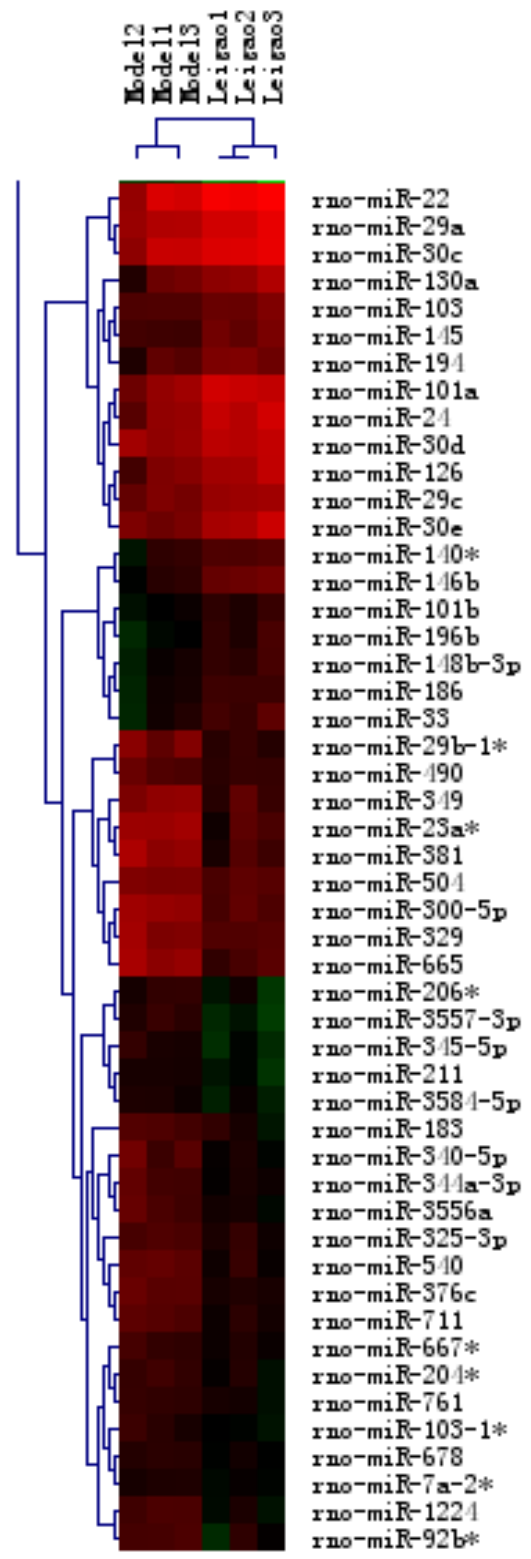

(a)

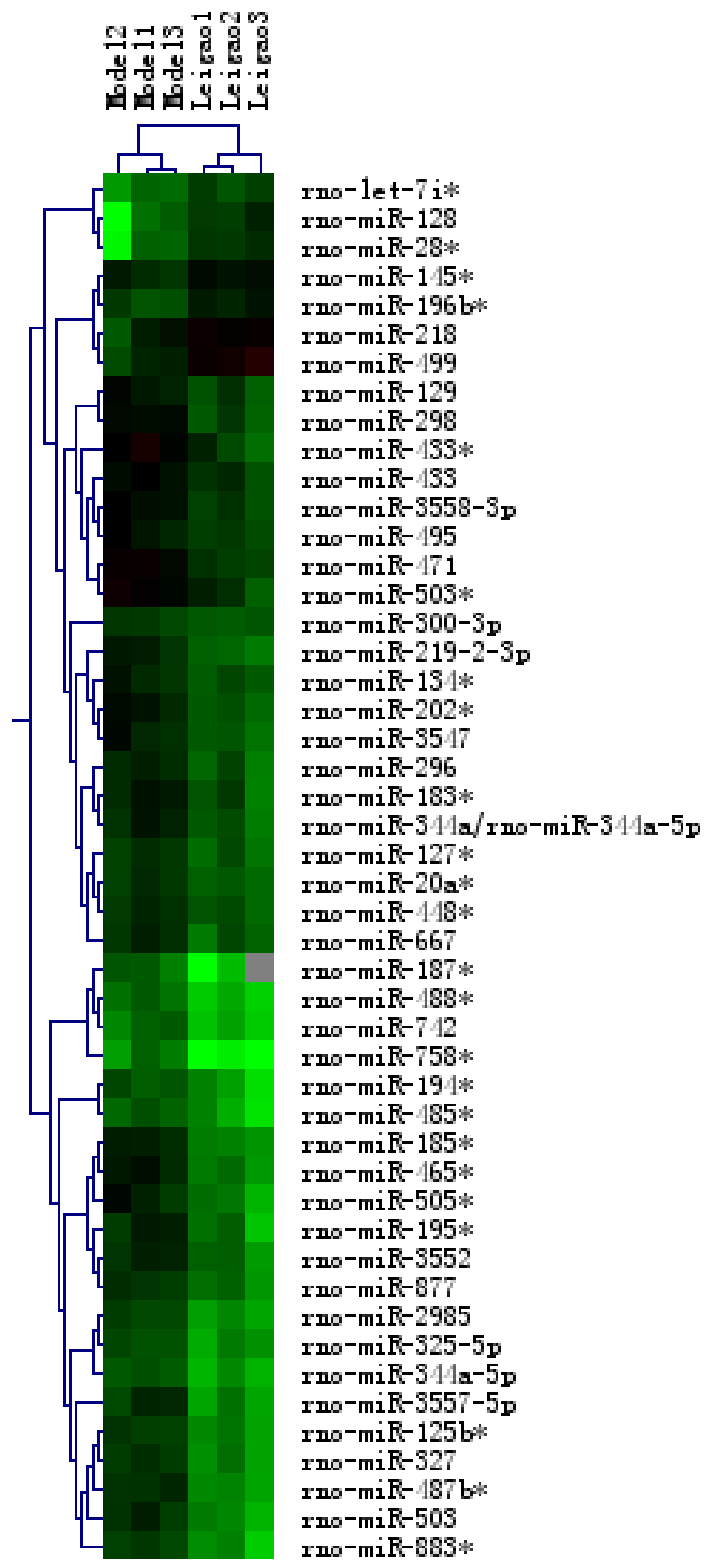

(b)

Figure 2. Differentially expressed miRNAs screening by miRNA Arrays. (a) Up-expressed miRNAs; (b) Down-expressed miRNAs. 
Dicer
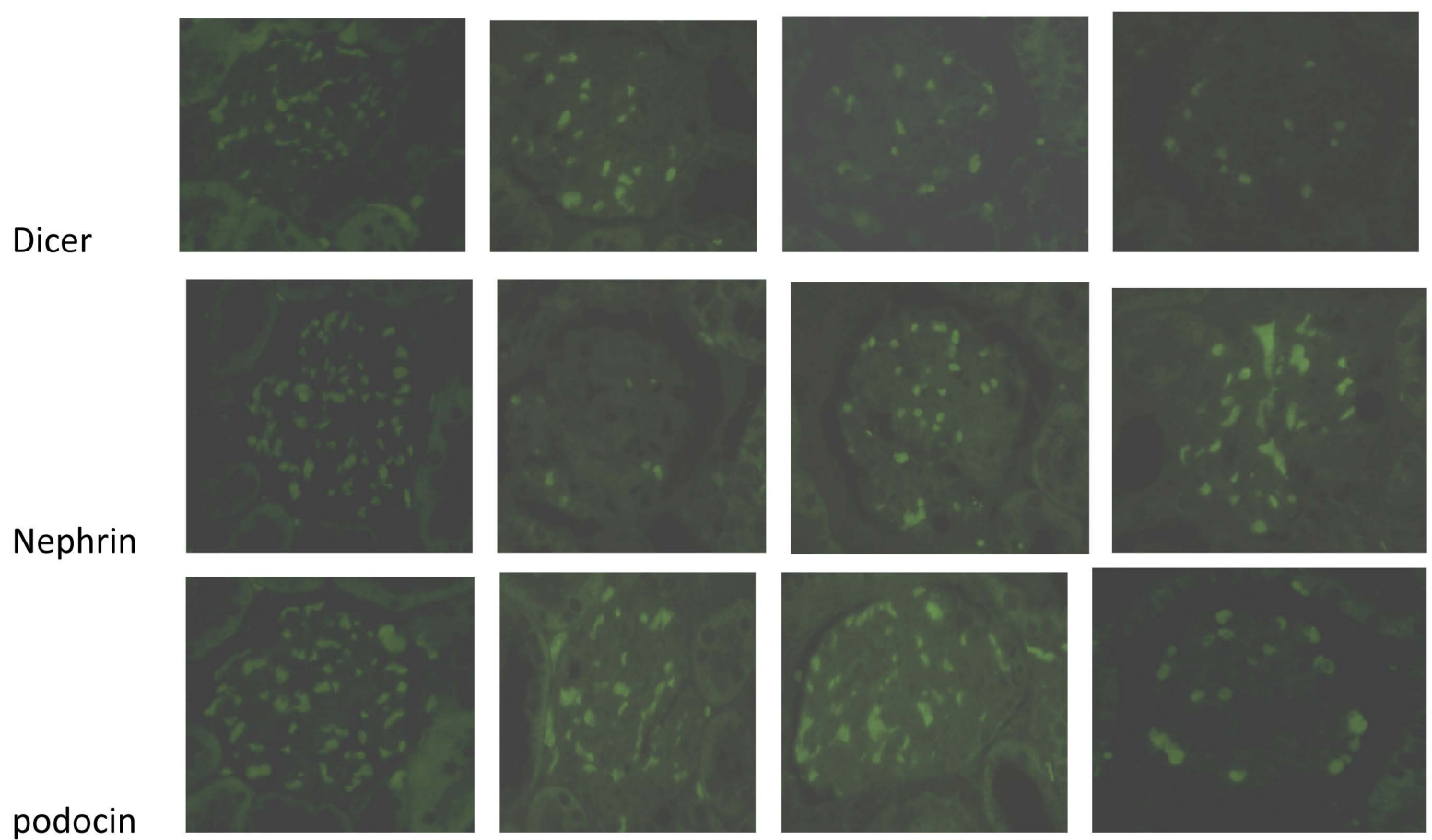

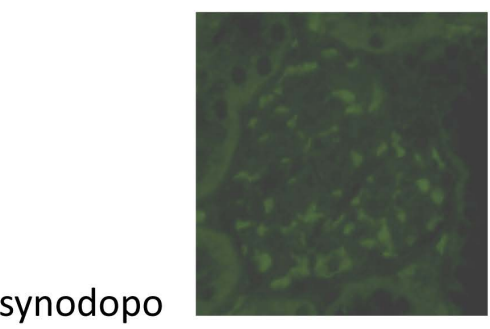

(a)

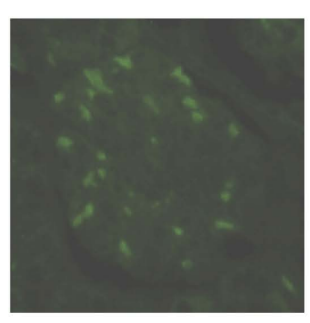

(b)

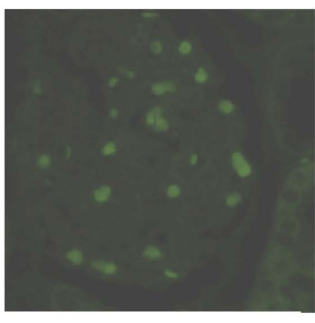

(c)

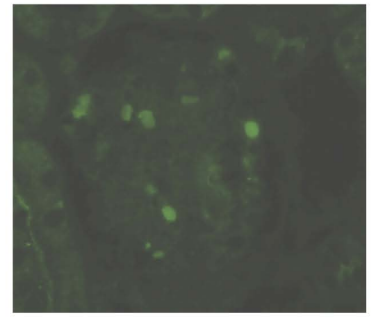

(d)

Figure 3. Immunoflunce of dicer, nephrin, podocin, and synodopotoptin in glomerular. (a) NS, (b) PAN, (c) Leizhi, (d) GTW.

\section{Discussion}

PAN nephrosis rats can be made successfully by jugular vein injection of PAN. PAN can selectively injury podocytes but not mesangium or endothelial cells in glomerular, inducing proteinuria, foot process effacement, and glomerular basement membrane abnormalities. So PAN nephrosis has been a classic podocyte-injury model.

Dicer is key to miRNA mature, which can mentain podocyte function and structure of SD in kidney. Our results show that dicer correlates well with proteinuria, but correlating negatively with nephrin and podocin. The most important finding in our study is the specific miRNA related to PAN nephrosis (rno-miR23a, rno-miR-24, rno-miR-30c and rno-miR-300-3p), which have been validated by Real time RT-PCR analysis. Up-regulated miRNAs (rno-miR-23a, rno-miR-300-3p) may trigger podocyte injury and proteinuria, while down-regulated miRNAs (rno-miR-24, rno-miR-30c) may be protective factors by anti-apoptosis. These miRNA may function corporately, because they located in the same chromesome [11] [12]. To explore dicer role in glomerular function, Harvey [8] et al. used a conditional Dicer allele to disrupt miRNA biogenesis in mouse podocytes. Mutant mice developed proteinuria and progressed rapidly to end-stage kidney disease. The pathology included foot process effacement, vacuolization, and hypertrophy with crescent formation. Their findings demonstrate a critical role for miRNA in glomerular function and suggest that podocyte may participate in the pathogenesis of kidney diseases. Shi [7] et al. inactivated dicer selectively in mouse podocytes. Mutant mice developed proteinuria 4 to 5 weeks after birth and died several weeks later, presumably from kidney failure. Multiple abnormal- 
ities including foot process effacement, irregular and split areas of the glomerular basement membrane, podocyte apoptosis and depletion, and glomerulosclerosis were observed. Four members of the mir-30 miRNA family were identified, known to be expressed and/or functional in podocytes. These results suggest functional roles for the mir-30 miRNA family in podocyte homeostasis and podocytopathies. Ho [9] et al. generated mice lacking functional miRNAs in the developing podocyte through podocyte-specific knockout of Dicer, Podocytespecific loss of miRNAs resulted in significant proteinuria, rapid progression of marked glomerular and tubular injury, and death. Expression of the slit diaphragm proteins nephrin and podocin was decreased, and expression of the transcription factor WT1 remained unchanged. To identify miRNA-mRNA interactions that contribute to this phenotype, they profiled the glomerular expression of miRNAs; three miRNAs expressed in glomeruli were identified: mmu-miR-23b, mmu-miR-24, and mmu-miR-26a. These results suggest that miRNA function is dispensable for the initial development of glomeruli but is critical to maintain the glomerular filtration barrier. Connect to the database of miRNA Base [13] [14], target genes of miR-24, miR-23a and miR-30c correlate with phosphatase phosphatkinase and Protease C, suggesting these miRNA may participate in nephrin phosphorylation in podocyte homeostasis and podocyte injury.

Dicer can be down-regulated in PAN induced rats treated with Tripterygium preparation. Expression profile of nephrin, podocin and synaptopodin mRNA and protein decrease in samples treated with Tripterygium wilfordii Hook by triggering dicer-miRNA, which play the podocyte protection role by inhibiting podocyte apotosis. So these miRNAs (rno-miR-24, rno-miR-30c, rno-miR-23a) and dicer may be target moculars of leizhi capsule on treating proteinuria in PAN nephrosis. They may be are probably key molecules therapeutic targets.

From our work, we can conclude that characteristic miRNAs of PAN nephrosis were screening. Up-regulated miRNAs (rno-miR-23a, rno-miR-300-3p) may trigger podocyte injury and proteinuria, while down-regulated miRNAs (rno-miR-24, rno-miR-30c) may be protective factors by anti-apoptosis. Dicer and these miRNAs (rnomiR-24, rno-miR-30c, rno-miR-23a) may be are probably key molecules therapeutic targets of Leizhi capsule. But the fine mechanisms remain to be clarified by more researches.

\section{Acknowledgements}

miRNA Array analysis has been accomplished by Shanghai Kang Chen Biological engineering company.

\section{Financial Support}

The experiment is funded by Jiangsu province natural science fund (BK2009462) and the Priority Academic Program Development of Jiangsu Higher Education Institutions (PAPD).

\section{Conflict of Interest Statement}

The authors declare no conflicts of interest.

\section{References}

[1] Lee, R.C., Feinbaum, R.L. and Ambros, V. (1993) The C. elegans Heterochronic Gene lin-4 Encodes Small RNAs with Antisense Complementarity to lin-14. Cell, 75, 843-854. http://dx.doi.org/10.1016/0092-8674(93)90529-Y

[2] Reinhart, B.J., Slack, F.J., Basson, M., et al. (2000) The 21-Nucleotide let-7 RNA Regulates Developmental Timing in Caenorhabditis elegans. Nature, 403, 901-906. http://dx.doi.org/10.1038/35002607

[3] Muljo, S.A., Kanellopoulou, C. and Aravind, L. (2010) MicroRNA Targeting in Mammalian Genomes: Genes and Mechanisms. Wiley Interdisciplinary Reviews: Systems Biology and Medicine, 2, 148-161. http://dx.doi.org/10.1002/wsbm.53

[4] Ohashi, T., Uchida, K., Asamiya, Y., et al. (2010) Phosphorylation Status of Nephrin in Human Membranous Nephropathy. Clinical and Experimental Nephrology, 14, 51-55. http://dx.doi.org/10.1007/s10157-009-0241-z

[5] Welsh, G.I. and Saleem, M.A. (2010) Nephrin-Signature Molecule of the Glomerular Podocyte? Journal of Pathology, 220, 328-337.

[6] Qin, X.S., Tsukaguchi, H., Shono, A., et al. (2009) Phosphorylation of Nephrin Triggers Its Internalization by RaftMediated Endocytosis. Journal of the American Society of Nephrology, 20, 2534-2545. http://dx.doi.org/10.1681/ASN.2009010011

[7] Shi, S., Yu, L., Chiu, C., et al. (2008) Podocyte-Selective Deletion of Dicer Induces Proteinuria and Glomerulosclero- 
sis. Journal of the American Society of Nephrology, 19, 2159-2169. http://dx.doi.org/10.1681/ASN.2008030312

[8] Harvey, S.J., Jarad, G., Cunningham, J., et al. (2008) Podocyte-Specific Deletion of Dicer Alters Cytoskeletal Dynamics and Causes Glomerular Disease. Journal of the American Society of Nephrology, 19, 2150-2158. http://dx.doi.org/10.1681/ASN.2008020233

[9] Ho, J., Ng, K.H., Rosen, S., et al. (2008) Podocyte-Specific Loss of Functional microRNAs Leads to Rapid Glomerular and Tubular Injury. Journal of the American Society of Nephrology, 19, 2069-2075. http://dx.doi.org/10.1681/ASN.2008020162

[10] Liu, L.H., Zhu, C.F. and Ou, L.Z. (2005) Optimization and Evaluation of Puromycin Amioenucleoside Nephropathy Model in Rats. Fudan University Journal of Medical Sciences, 32, 488-492.

[11] Chhabra, R., Dubey, R. and Saini, N. (2010) Cooperative and Individualistic Functions of the microRNAs in the miR23a 27a 24-2 Cluster and Its Implication in Human Diseases. Molecular Cancer, 9, 232. http://dx.doi.org/10.1186/1476-4598-9-232

[12] Chhabra, R., Adlakha, Y.K., Hariharan, M., et al. (2009) Upregulation of miR-23a-27a-24-2 Cluster Induces Caspase-Dependent and -Independent Apoptosis in Human Embryonic Kidney Cells. PLoS One, 4, e5848. http://dx.doi.org/10.1371/journal.pone.0005848

[13] Griffiths-Jones, S. (2010) miRBase: microRNA Sequences and Annotation. Current Protocols in Bioinformatics, 12, 12-19.

[14] Griffiths-Jones, S., Grocock, R.J., van Dongen, S., et al. (2006) miRBase: microRNA Sequences, Targets and Gene Nomenclature. Nucleic Acids Research, 34, D140-D144. http://dx.doi.org/10.1093/nar/gkj112 
Scientific Research Publishing (SCIRP) is one of the largest Open Access journal publishers. It is currently publishing more than 200 open access, online, peer-reviewed journals covering a wide range of academic disciplines. SCIRP serves the worldwide academic communities and contributes to the progress and application of science with its publication.

Other selected journals from SCIRP are listed as below. Submit your manuscript to us via either submit@scirp.org or Online Submission Portal.
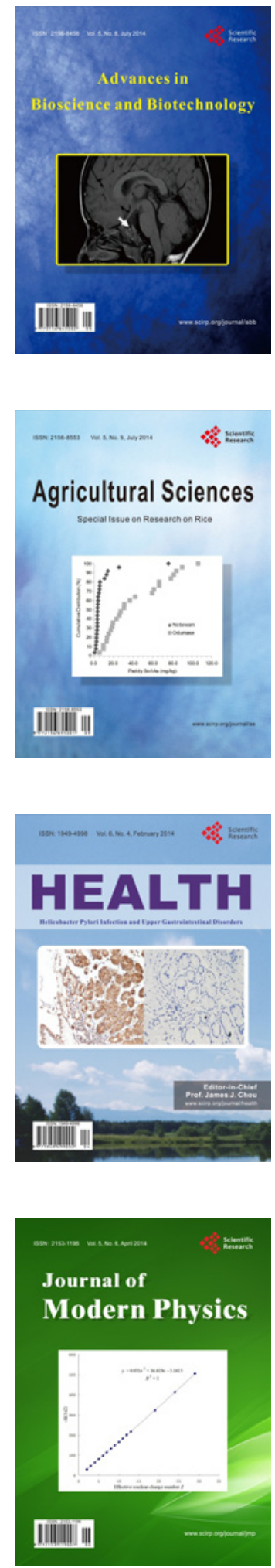
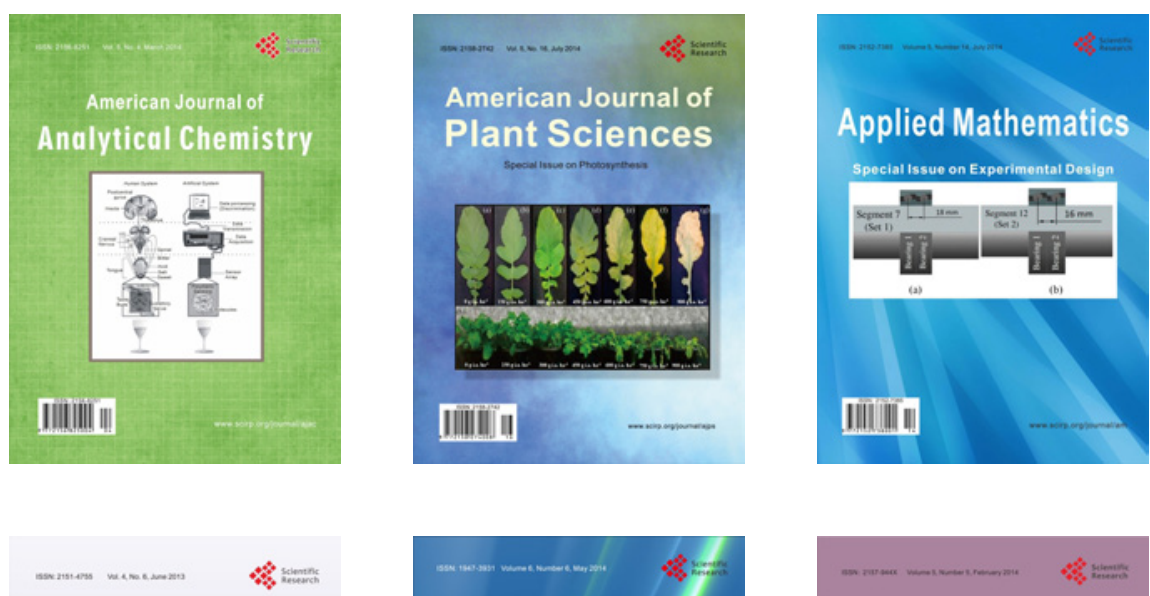

Creative Education
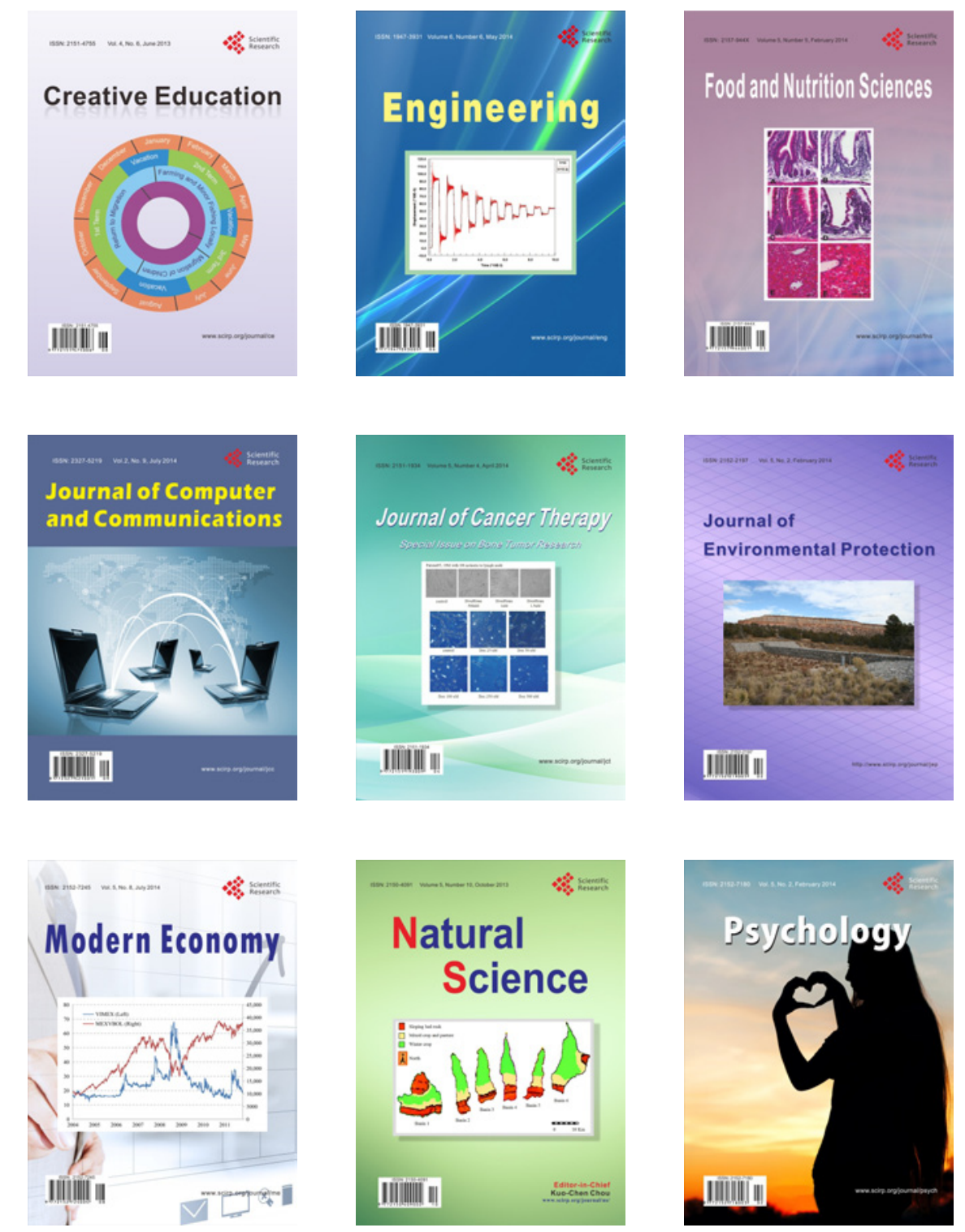